\title{
BMJ Open Protocol of a 12-month multifactorial eHealth programme targeting balance, dual-tasking and mood to prevent falls in older people: the StandingTall+ randomised controlled trial
}

Kimberley S van Schooten, ${ }^{1,2}$ Michele L Callisaya, ${ }^{3,4}$ Bridianne O'Dea, ${ }^{5}$ Thomas Lung (1) ,6,7 Kaarin Anstey, ${ }^{1,8}$ Stephen R Lord, ${ }^{1,2}$ Helen Christensen, ${ }^{5}$ Alicia Brown, ${ }^{1}$ Jessica Chow, ${ }^{1}$ Garth McInerney, ${ }^{1}$ Lillian Miles, ${ }^{1}$ Michelle Ngo, ${ }^{1}$ Amy Perram, ${ }^{1}$ Kim Delbaere (i) ${ }^{1,2}$

To cite: van Schooten KS, Callisaya ML, O'Dea B, et al. Protocol of a 12-month multifactorial eHealth programme targeting balance, dual-tasking and mood to prevent falls in older people: the StandingTall+ randomised controlled trial. BMJ Open 2021;11:e051085. doi:10.1136/ bmjopen-2021-051085

- Prepublication history and additional supplemental material for this paper are available online. To view these files, please visit the journal online (http://dx.doi.org/10.1136/ bmjopen-2021-051085).

Received 10 March 2021 Revised 21 March 2021 Accepted 23 March 2021

Check for updates

(C) Author(s) (or their employer(s)) 2021. Re-use permitted under CC BY-NC. No commercial re-use. See rights and permissions. Published by BMJ.

For numbered affiliations see end of article.

Correspondence to

Dr Kim Delbaere;

k.delbaere@neura.edu.au

\section{ABSTRACT}

Introduction Falls have a multifactorial aetiology, which may limit the effectiveness of the common approach of exercise as the sole intervention strategy. Multifactorial interventions could be more effective in people at high risk of falling; however, the focus of such interventions has traditionally been quite narrow. This paper describes the design of a randomised controlled trial that will evaluate the effectiveness of an eHealth programme, which addresses cumulative effects of key fall-risk factors across the triad of physical, affective and cognitive functions on falls in older people.

Methods and analysis 518 older people aged 65 years and over with high fall risk, defined as having a history of falls in the past 6 months, self-reported fear of falling or being aged 80 years or over, will be recruited via local advertisements, newsletters and presentations, and randomised to an intervention or health education control group. The intervention comprises balance exercise, cognitive-motor exercise and cognitive-behavioural therapy, with their dosage based on participant's baseline balance, executive function and mood. The primary outcome is the rate of falls in the 12 months after randomisation. Secondary outcomes at 6 and 12 months comprise programme adherence, healthcare use, physical activity, balance and mobility, cognitive function, psychological well-being, quality of life, health literacy and user experience and attitudes towards the programme. Data will be analysed following intention to treat to gauge real-world effectiveness. We will further determine complier averaged causal effects to correct for varying adherence and conduct economic analyses to gain insight into cost-effectiveness and cost-utility. Ethics and dissemination Ethical approval was obtained from the University of New South Wales (UNSW) Human Research Ethics Committee in December 2017. Outcomes will be disseminated via peer-reviewed articles, conference presentations, community events and media releases.

\section{Strengths and limitations of this study}

- The randomised controlled design provides a rigorous means to investigate the effectiveness of a tailored eHealth programme on reducing fall rates in older people.

- The multifactorial programme addresses balance, dual-tasking ability and mood, targeting important fall-risk factors.

- The use of technology allows for remote and personalised intervention with intensity progression over time.

- COVID-19 has required us to switch to telehealth for (re)assessments mid-trial, which will likely increase noise and reduce statistical power for secondary outcomes.

- Our statistical analysis is not powered to disentangle programme component effectiveness on our primary outcome of fall rates.

Trial registration number ACTRN12619000540112.

\section{INTRODUCTION}

Effective and scalable strategies for fall prevention are essential to support the healthcare system. With ageing population, falls and falls injuries are increasing and currently available fall prevention programmes are not able to curb the incidence at a public health level. eHealth provides an opportunity to deliver fall prevention interventions that are highly scalable at relatively low costs. These technology-supported interventions have the advantage that they overcome common barriers to adherence, such as being intimidated by group settings, lack of motivation to attend a session and organisational barriers of location accessibility 
and caring responsibilities. ${ }^{1}$ Previous studies have shown that eHealth is acceptable and feasible for older people, with high average dose adherence rates of up to $140 \%$ $\left(\mathrm{eg},{ }^{23}\right)$. Our recent study, using the eHealth balance exercise intervention StandingTall, showed that $52 \%$ of older participants achieved an average weekly dose of $120.3 \mathrm{~min}$ that was sustained over a 2-year intervention period. ${ }^{4}$ The StandingTall eHealth intervention reduced fall rates by $16 \%$ and reduced the proportion of people experiencing injurious falls by $20 \%{ }^{4}$

Falls are often caused by a reduced balance control which is known to gradually decline with advancing age. ${ }^{5}$ However, psychological distress and decline of cognitive abilities, especially executive function, can also contribute to falls in older people independent of poor balance. ${ }^{67}$ It has been shown that a marked deficiency in just one domain is sufficient to increase fall risk. ${ }^{8}$ However, multiple deficiencies of only moderate severity add cumulatively and their summation can also increase fall risk. ${ }^{9}$ Research has shown that older people have an increased reliance on cognitive control while walking and doing their daily activities, as partial compensation for age-related changes in physical function. ${ }^{10}$ Therefore, in combination with poor balance, even mild cognitive impairment can result in an increased risk of falling. ${ }^{11}$ Similarly, psychological distress competes for resources in working memory, and might interfere with tasks requiring complex attention and coordination, ${ }^{12}$ which can then result in an increased risk of falls. ${ }^{13}$ Concomitant deficiencies across physical, affective and cognitive domains are more common in old age, resulting in an exponential increase in fall risk as people age.

The multifactorial aetiology of falls warrants a multifactorial intervention, but improvements in focus and delivery are needed. Common strategies in multifactorial programmes overlook the cognitive and affective risk domains that contribute to falls in older people. Perhaps because of this, evidence of their effectiveness is inconclusive and effect sizes are moderate at a $23 \%$ reduction of fall rates. ${ }^{14}$ Dual-task exercise and cognitive-behavioural therapy (CBT) address cognitive and affective domains and have been shown to reduce falls or fall risk factors in people with high fall risk. ${ }^{1516}$ Moreover, multifactorial interventions should be carefully targeted to avoid unnecessary efforts and costs. ${ }^{17}$ Therefore, we will evaluate an individually tailored approach addressing physical, affective and cognitive domains with balance exercise, cognitive-motor and CBT to reduce falls in high-risk older people. We will use eHealth to optimise the delivery of the different intervention components and maximise adherence. This will allow us to offer a complex intervention programme that is easy to use, with high flexibility and other benefits to overcome many of the barriers to uptake and adherence identified in previous multifactorial research trials.

This paper describes the protocol of a randomised controlled trial in community-dwelling older people at high risk of falling (defined as falls in the past 6 months, fear of falling or aged $80+$ years). The primary aim is to test the hypothesis that a tailored, multifactorial eHealth programme will lead to a lower incidence of falls over a 12-month period, when compared with a health promotion programme. The secondary aims are to determine the efficacy of the programme on known (physical, affective, cognitive) fall-risk factors and to calculate the costeffectiveness of the intervention.

\section{METHODS AND ANALYSIS \\ Design}

We will conduct a two-arm, assessor-blinded randomised controlled trial to examine the effectiveness of our tailored StandingTallt programme over 1 year. Recruitment started in June 2019 and the trial is expected to finish by December 2021. Both intervention and control groups will receive a health education programme, in addition to usual care. Our primary outcome will be rate of falls over 1 year, with secondary outcomes of programme adherence, healthcare use, physical activity, balance and mobility, cognitive function, psychological well-being, quality of life, health literacy and user experience and attitudes towards the programme. The trial protocol has been registered prior to starting on the Australian and New Zealand Clinical Trial Registry (ACTRN12619000540112; summary in figure 1, table 1).

\section{Participants}

We will recruit 518 older people at high risk for falls based on the following self-reported criteria: experienced $\geq 1$ falls in the past 6 months or have a self-reported fear of falling or are aged 80 years or over. Participants will all be aged 65 years or over, living in the community, proficient in English, independent in activities of daily living, able to walk household distances (defined as $10 \mathrm{~m}$ ) without the use of a walking aid, willing to provide informed consent and comply with the study protocol (all self-reported). Exclusion criteria (self-reported except if specified) are having an unstable or acute medical condition that precludes exercise participation, progressive neurological condition, cognitive impairment (determined by a Short Portable Mental Status Questionnaire score $<8,{ }^{18}$ severe depression or suicidal thoughts (determined by a Patient Health Questionnaire-9 score $\geq 20$ or a score of 3 on item $9,{ }^{19}$ acute psychiatric condition with psychosis, or are currently participating in a fall prevention programme.

\section{Recruitment}

Participants will be recruited primarily through presentation of the study to senior audiences, sourced via contacts at independent living facilities, advertisements in community services newsletters, notice boards, local newspapers, websites and social media. People who are interested in the study will call our automatic voice message system or visit our website and leave their contact details. They will receive a call back during which we provide information about the study and screen for eligibility after a verbal 
Recruitment of community-dwelling older people at high risk of falls

\section{Screening}

Baseline assessment

Concealed allocation $(\mathrm{N}=518)$

Standing Tall+ intervention group $(\mathrm{N}=259)$

Health education control group $(\mathrm{N}=259)$

Access to health education website

StandingTall+ (home/telehealth) set-up

Follow-up at 4 weeks

Follow-up and reassessments

Continuous monitoring of falls (primary outcome) and of adverse events, program adherence, health care use

Reassessment at 6 months of physical activity, balance and mobility; cognitive function; psychological well-being; quality of life; health literacy; (intervention group only) user experience and attitude towards the program

Reassessment at 12 months of physical activity; cognitive function; psychological well-being; quality of life; health literacy; self-reported change; (intervention group only) user experience and attitude towards the program

Figure 1 Study design.

consent. If screening criteria are met and the person is interested in participating in the study, they will receive the participant information statement and consent form via email. An appointment with study personnel for signing the informed consent (provided as online supplemental file 1) and the baseline assessment will be made during a second call, allowing for time to read the participant information statement and consent form.

\section{Group allocation}

Participants will be randomly allocated to the intervention or health education control group after the baseline assessment. Permuted block randomisation (1:1 allocation ratio) will be performed using a custom randomisation programme. People living in the same household will be allocated together to prevent contamination, and randomised in their own blocks to ensure that both groups have an equal number of couples. Allocation will be concealed until 1 week after baseline to ensure blinding of the assessors (table 2). Assessors will be blinded throughout the study; participants will be aware of their group allocation but are reminded to not disclose this during assessments by blinded assessors. Unblinded study personnel are available to help with setting up the programme and answer any study-related queries.

\section{Intervention components}

The StandingTall+ intervention comprises 12 months of tailored balance exercise, CBT and cognitive-motor training (CMT). The intervention group will receive up to three programmes depending on their baseline status of balance, executive function and mood (table 3). All programmes are delivered through a tablet computer (provided by the research team if necessary) and are complemented by personalised encouragement messages and compliance-promoting features.

\section{StandingTall balance exercise}

All intervention participants will receive the StandingTall balance exercise programme starting from week 1 to week 52, and 10 monthly fact sheets on the importance of an active and healthy lifestyle. The home-based exercise training offers an effective way for people to reduce their fall risk, improve balance and increase quality of life. ${ }^{4}$ 


\begin{tabular}{|c|c|}
\hline Category & Information \\
\hline Trail registry & anzctr.org.au: ACTRN12619000540112 \\
\hline $\begin{array}{l}\text { Registration } \\
\text { date }\end{array}$ & 4 April 2019 \\
\hline $\begin{array}{l}\text { Source of } \\
\text { support }\end{array}$ & NHMRC grant: APP1139673 \\
\hline Primary sponsor & $\begin{array}{l}\text { Neuroscience Research Australia, contact: } \\
\text { d.mckay@neura.edu.au }\end{array}$ \\
\hline $\begin{array}{l}\text { Public/scientific } \\
\text { queries }\end{array}$ & Profesor Delbaere, k.delbaere@neura.edu.au \\
\hline Public title & $\begin{array}{l}\text { StandingTall-Plus: A 1-year randomised } \\
\text { controlled trial of a novel multifactorial } \\
\text { intervention for preventing falls in older } \\
\text { people }\end{array}$ \\
\hline Scientific title & $\begin{array}{l}\text { A novel multifactorial intervention for preventing } \\
\text { falls in older people over } 1 \text { year: randomised } \\
\text { controlled trial }\end{array}$ \\
\hline $\begin{array}{l}\text { Country of } \\
\text { recruitment }\end{array}$ & Australia \\
\hline $\begin{array}{l}\text { Health problem } \\
\text { studied }\end{array}$ & Accidental falls \\
\hline Intervention & $\begin{array}{l}\text { Active comparator: Multifactorial tailored } \\
\text { eHealth intervention including a home- } \\
\text { based balance exercise programme } \\
\text { (StandingTall), CBT (myCompass), and/or CMT } \\
\text { (StandingTall+Cognitive) }\end{array}$ \\
\hline
\end{tabular}

Control comparator: Health promotion education programme

$\begin{array}{ll}\begin{array}{l}\text { Inclusion/ } \\ \text { exclusion criteria }\end{array} & \text { Ages eligible for study: } \geq 65 \text { years; sexes eligible: } \\ & \text { Healthy volunteers at high risk of falls: a history of } \\ \text { falls in the past } 6 \text { months or self-reported fear of } \\ \text { falling or } 80+\text { years } \\ \text { Inclusion criteria: } 65 \text { years old or over, living in the } \\ \text { community, proficient in English, independent in } \\ \text { activities of daily living, able to walk household } \\ \text { distances without the use of a walking aid, and } \\ \text { willing to give informed consent and comply with } \\ \text { the study protocol } \\ \text { Exclusion criteria: unstable or acute medical } \\ \text { condition precluding exercise, progressive } \\ \text { neurological condition, cognitive impairment } \\ \text { (SPMSQ <8), severe depressive symptoms or } \\ \text { suicidal thoughts (PHQ-9 } \geq 20 \text { or score of } 3 \text { on } \\ \text { last PHQ-9 item) or current participation in fall } \\ \text { prevention programme }\end{array}$

Continued

\begin{tabular}{ll} 
Table 1 & Continued \\
\hline Category & Information \\
\hline $\begin{array}{l}\text { Key secondary } \\
\text { outcomes }\end{array}$ & $\begin{array}{l}\text { Proportion fallers/multiple fallers in each group, } \\
\text { programme adherence, healthcare use, physical } \\
\text { activity, balance and mobility, cognitive function, } \\
\text { psychological well-being, quality of life, health } \\
\text { literacy and user experience and attitudes } \\
\text { towards the programme }\end{array}$
\end{tabular}

ACTRN, Australian and New Zealand Clinical Trial Registry; CBT, cognitive-behavioural therapy; CMT, cognitive-motor therapy; NHMRC, National Health and Medical Research Council; PHQ-9, Patient Health Questionnaire-9; SPMSQ, Short Portable Mental Status Questionnaire.

It comprises standing balance (eg, standing on a foam surface), transferring (eg, sit-stand transitions), walking (eg, walking in circles or to targets in a grid), stepping (eg, step and lift) and box (eg, step up and over a box) exercises (figure 2). The programme is fully optimised to deliver unsupervised and individually tailored balance exercises that increase in difficulty over time. Participants will be instructed to complete $40 \mathrm{~min} /$ week of exercise in the first 2 weeks. This weekly exercise dosage will increase by 20 min fortnightly, to finally reach a dose of 2 or 3 hour/ week. This final dosage of 2 or 3 hour/week will be based on their baseline assessment of executive function and balance. Participants with intact executive functioning (defined as a trail making test part B minus part A duration $<50 \mathrm{~s},{ }^{9}$ who have a poor balance (defined as a Physiological Profile Assessment score $>0.6^{9}$ or, if this score is not available, tandem stance duration $<30 \mathrm{~s}^{20}$ will be asked to adhere to a dose of 3 hours per week. All others will be asked to adhere to a dose of 2 hours per week. Participants have full autonomy to choose the timing and duration of their sessions to achieve their weekly dose.

\section{StandingTall+Cognitive CMT}

Intervention participants with poor executive functioning (defined as a baseline trail making test part B minus part A duration $>50 \mathrm{~s}^{9}$ will receive the StandingTall+Cognitive CMT programme to improve executive function and attention from week 5 to week 52 . Concomitant cognitive tasks, relying primarily on executive function, are added to the StandingTall balance exercises by using auditory and visual cues (figure 2). Three core executive functions are engaged: inhibition (the ability to consciously override automated or dominant responses), working memory (the ability to hold, process and manipulate information in mind) and task shifting (the ability to switch flexibly between tasks or mental sets). Participants will receive $50 \%$ (if on a 2-hour StandingTall dose) or 33\% (if on a 3-hour StandingTall dose) of their weekly StandingTall balance exercise dose, with a final dose of 1 hour per week, as StandingTall+Cognitive programme.

\section{MyCompass CBT}

Intervention participants with depressive symptoms (defined as a baseline 15-item geriatric depression scale 
Table 2 List of measurements and time points

\begin{tabular}{|c|c|c|c|c|c|c|c|c|}
\hline & \multicolumn{2}{|l|}{ Enrolment } & \multirow{2}{*}{$\begin{array}{l}\text { Allocation } \\
\text { Oweek }\end{array}$} & \multicolumn{5}{|c|}{ Post-allocation } \\
\hline & $<-1$ week & -1 week & & 1 week & 5 weeks & 7 weeks & 26 weeks & 52 weeks \\
\hline \multicolumn{9}{|l|}{ Enrolment } \\
\hline $\begin{array}{l}\text { Short Portable Mental Status } \\
\text { Questionnaire }\end{array}$ & $x$ & & & & & & & \\
\hline $\begin{array}{l}\text { Sociodemographic } \\
\text { characteristics }\end{array}$ & $\mathrm{x}$ & & & & & & & \\
\hline Informed consent & & $x$ & & & & & & \\
\hline Allocation & & & $x$ & & & & & \\
\hline \multicolumn{9}{|l|}{ Interventions } \\
\hline Health Education Programme (all) & & & & $<------$ & ---------. & ------------ & ------------ & ----------> \\
\hline $\begin{array}{l}\text { StandingTall Balance Exercise } \\
\text { Programme (intervention) }\end{array}$ & & & & $<-------$ & ------- & ----------- & -----------.- & ----------> \\
\hline
\end{tabular}

\section{Assessments}

\section{Descriptive characteristics}

Age, gender, education, livingsituation, fall history

Charlson Comorbidity Index X

NEO-FFI Neuroticism and Openness $\quad X$

Montreal Cognitive Assessment $\quad X$

Spot the Word

Falls, adverse events, adherence and costs

Falls and adverse events

Adverse events

\section{Adherence}

Healthcare use-questionnaire

Healthcare use-linked data

Intervention costs

$x$
$x$
$x$

Physical activity, balance and mobility

\begin{tabular}{|c|c|c|c|}
\hline $\begin{array}{l}\text { Incidental and Planned Exercise } \\
\text { Questionnaire }\end{array}$ & $\mathrm{x}$ & $x$ & $\mathrm{x}$ \\
\hline 1 week of mobility monitoring & $\mathrm{x}$ & $\mathrm{x}$ & $\mathrm{x}$ \\
\hline Physiological Profile Assessment ${ }^{\star}$ & $\mathrm{X}$ & $x$ & \\
\hline $\begin{array}{l}\text { Standing balance with feet in } \\
\text { different positions }\end{array}$ & $\mathrm{X}$ & $\mathrm{X}$ & \\
\hline Maximum anteroposterior lean test* & $\mathrm{X}$ & $x$ & \\
\hline Coordinated stability test* & $x$ & $\mathrm{x}$ & \\
\hline Short Physical Performance Battery & $\mathrm{x}$ & $x$ & \\
\hline Timed up-and-go test & $\mathrm{x}$ & $\mathrm{x}$ & \\
\hline Five time sit-to-stand test & $x$ & $x$ & \\
\hline $\begin{array}{l}\text { Timed } 10 \mathrm{~m} \text { walk test ( } 4 \mathrm{~m} \text { walk via } \\
\text { telehealth) }\end{array}$ & $\mathrm{X}$ & $x$ & \\
\hline $\begin{array}{l}\text { Timed } 10 \mathrm{~m} \text { walk test with cognitive } \\
\text { dual task }\end{array}$ & $\mathrm{x}$ & $\mathrm{x}$ & \\
\hline $\begin{array}{l}\text { Patient's Global Impression of } \\
\text { Change Scales }\end{array}$ & & $x$ & $\mathrm{X}$ \\
\hline
\end{tabular}


Table 2 Continued

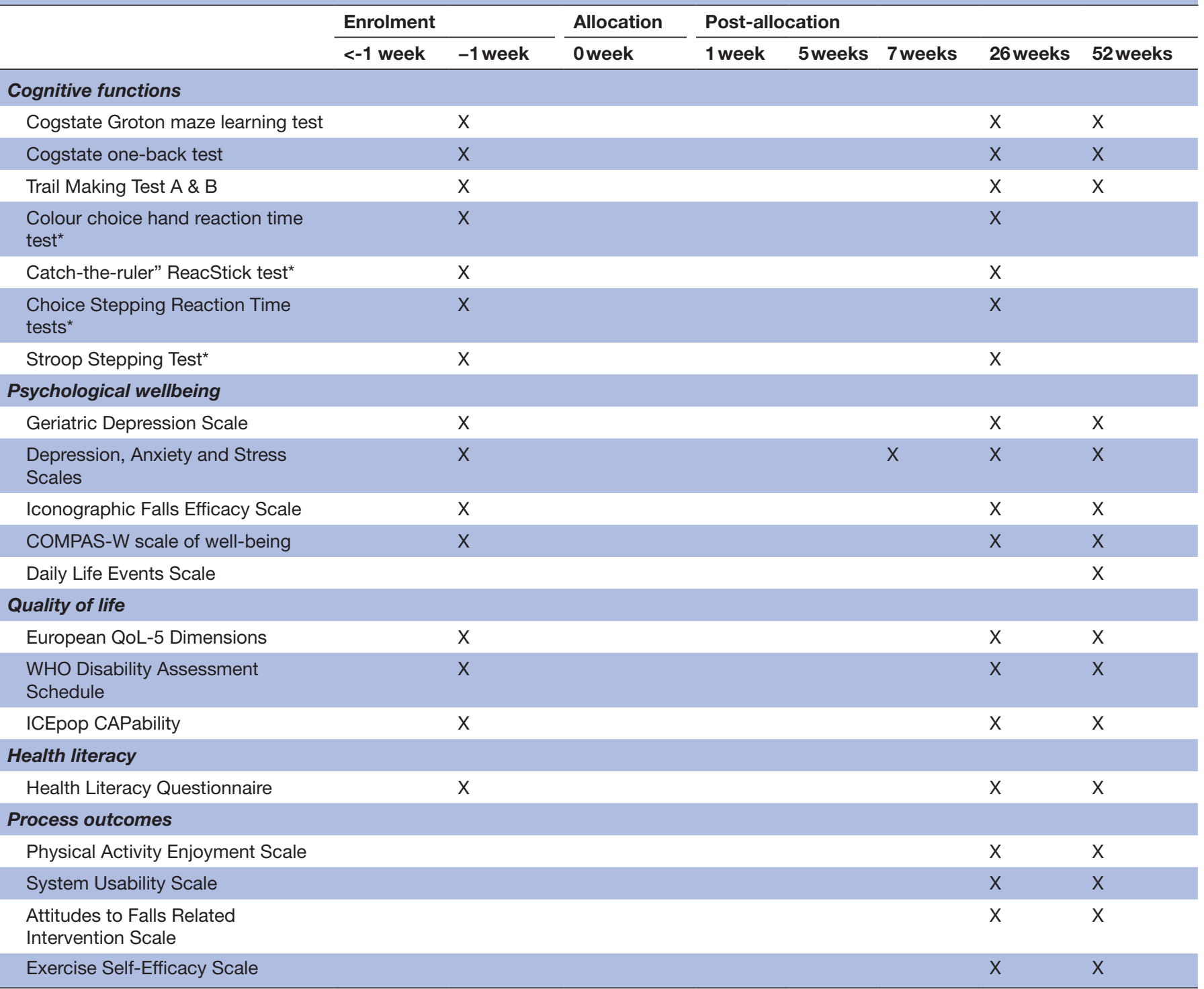

*Indicates tests that are not available for assessments via telehealth.

CBT, cognitive-behavioural therapy; CMT, cognitive-motor therapy.

score $>5^{6}$ will receive the myCompass automated web-based CBT programme from week 1 to week 7 . The myCompass programme offers self-directed, evidence-based and interactive psychological modules that users can complete at any time and on any internet-enabled tablet or computer. ${ }^{21}$ Each module comprises three $10 \mathrm{~min}$ educational sessions combined with activities. Users are asked to undertake home practice tasks in between their online sessions (ie, completion of one full module per week followed by 1 week of practice), which are intended

Table 3 Intervention personalisation

\begin{tabular}{|c|c|c|c|}
\hline Intervention & Dose & Who & Focus \\
\hline StandingTall & 2 hours/week & All & Balance \\
\hline StandingTall & add 1 hour/week & $\begin{array}{l}\text { TMT } B-A<50 s \text { and } \\
(\text { PPA } \geq 0.6 \text { points OR tandem stance }<30 \text { s) }\end{array}$ & Balance \\
\hline StandingTall+Cognitive & add 1 hour/week & TMT $B-A \geq 50 s$ & Dual tasking \\
\hline MyCompass & add $30 \mathrm{~min} /$ week for 7 weeks & GDS- $15 \leq 15$ points & Mood \\
\hline
\end{tabular}

*Changed in March 2019 as we switched to telehealth assessments due to COVID-19.

GDS-15, 15-item Geriatric Depression Scale; PPA, Physiological Profile Assessment; TMT B-A, Trail Making Test B minus A. 


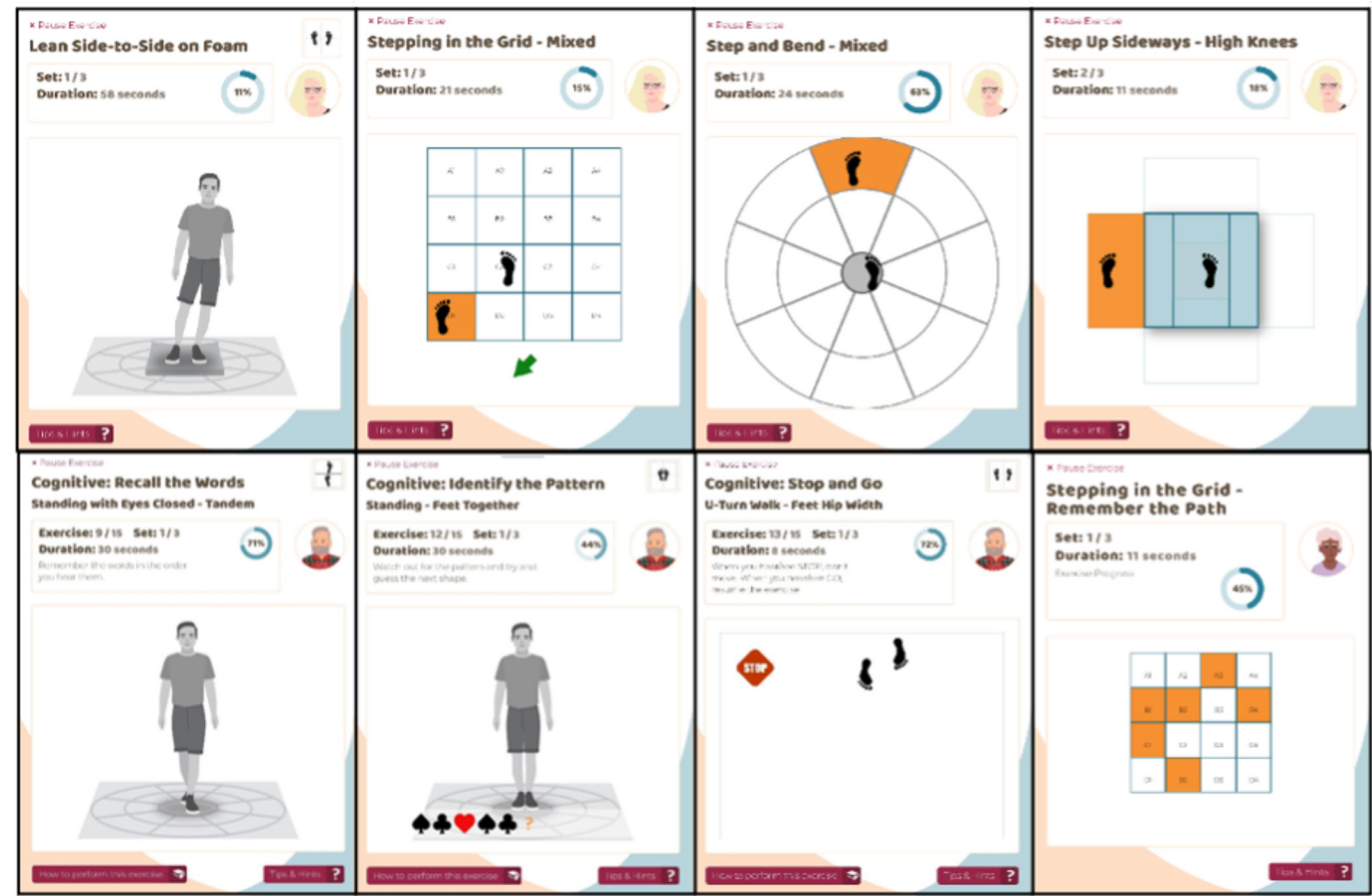

Figure 2 Examples of exercises from the StandingTall (top row; floor, grid, dartboard, step box exercises) and StandingTall+Cognitive programme (bottom row; attention, pattern recognition, inhibition, memory exercises).

to promote skill generalisation. In the current study, participants will be instructed to complete one module per week and self-monitor their behaviour daily (total of 30 min per week), with the aim of completing three full modules during the first 7 weeks of the trial.

Health promotion education control programme

All participants will receive the health promotion education programme. This programme comprises 52 weekly factsheets with a focus on general health concerns in older people (eg, blood pressure, healthy diet and medication). The programme will be provided to participants through a website with weekly email updates. Participants are set up with the programme during their group allocation call, 1 week after baseline. Tablets will be provided to all participants, with $4 \mathrm{G}$ connectivity for those without internet connection, to access the programmes and respond to questionnaires.

\section{Onboarding}

The intervention group will additionally receive a 1.5 hours onboarding session during which a trained exercise physiologist will explain the intervention and its relevant programme components. The exercise specialist will conduct StandingTall's inbuilt balance assessment (a composite score of standing balance with feet in different positions, which is repeated monthly) to ensure that the exercise difficulty level is appropriate for the participant's abilities. They will further explain the exercise and safety instructions, scheduling, tracking and goal setting features, and help the participant access the myCompass programme if appropriate.

\section{Adherence promotion and technical support}

All participants will receive a semi-structured phone call around week 4 as reminder of the health promotion education programme and, when relevant, follow-up on adherence. If the participant is in the intervention programme, and has poor executive functioning, the phone call around week 4 will also serve as a reminder that the StandingTall+Cognitive programme will become available as of week 5. During the first 6 months, participants not achieving their programme dose for two consecutive weeks will be contacted by telephone to discuss any issues that have arisen, and to encourage adherence. Phone, email and in-person/telehealth support will be available as needed for the entire duration of the study. The frequency and duration of this support will be registered for our economic evaluations.

\section{Assessments and impact of COVID-19}

All outcome measures will be obtained by trained exercise physiologists or physiotherapists blinded to group allocation. Due to the COVID-19 pandemic, all recruitment efforts, assessments, onboarding and other participant contacts were moved online using telehealth. Participants enrolled before March 2020 were seen in-person at our offices at Neuroscience Research Australia (NeuRA) for their assessments, and at home for the onboarding procedure. Participants enrolled after March 2020, and where 
possible any follow-up assessments for existing participants, will be seen via encrypted teleconferencing software. Where this is impracticable because of participant's home situations or access to technology, we will organise a face-to-face visit to our laboratory abiding to all applicable COVID-19 safety procedures. This change renders it unfeasible or unsafe to obtain some of our secondary outcomes, as detailed in the outcomes section below.

\section{Sample size calculation}

A sample size calculation using custom Stata code with $\mathrm{p}<0.05$, power of 0.8 and $20 \%$ drop-out rate ${ }^{4}$ indicates that a total sample size of 518 will be necessary to see an effect on the fall rate. An $\alpha$ value (measure of overdispersion in negative binomial regression) of 1.2 was taken from a previous fall prevention trial. ${ }^{22}$ The control group fall rate was assumed to be 1.098 falls per personyear. $^{9}$ An incidence rate ratio of 0.67 was chosen as the smallest worthwhile effect that justifies associated costs, risks and inconveniences, ${ }^{23}$ which was also supported by meta-regression results for exercise ${ }^{24}$ and multifactorial interventions. $^{25}$

\section{Outcomes}

\section{Primary outcome}

Our primary outcome is the rate of falls (expressed as the number of falls per person-year) in the 12 months after randomisation assessed by monthly digital fall calendars and confirmed via telephone contact. A fall is defined as an 'event that resulted in a person coming to rest unintentionally on the ground or other lower level' ${ }^{26}$ Participants are instructed that this definition includes trips and slips in which they lost their balance and landed on the floor or ground or lower level. We further obtain information on the location of the fall, activity at time of the fall, alleged cause of the fall, and any injuries and medical visits that resulted as consequence of the fall.

\section{Descriptive characteristics}

Potential covariates for secondary analyses are weight, height, age, gender, years of education, living situation, fall history, the Charlson Comorbidity Index,${ }^{27}$ NEO-FFI Neuroticism and Openness questionnaire, ${ }^{28}$ Montreal Cognitive Assessment ${ }^{29}$ and Spot the Word test. ${ }^{30}$

\section{Secondary outcomes}

Secondary outcomes span the proportion of fallers, and assessments across sensorimotor, cognitive and affective domains (see table 2 for an overview and timings). These outcomes are obtained at baseline and 6 months, and a short version is obtained at 12 months.

Fallers: Proportion of fallers, multiple fallers and injurious falls over 12 months. Injurious falls are defined as falls resulting in any injury (eg, bruises, cuts/grazes, joint dislocations, sprains/strains, fractures, pain), or falls that required medical care (eg, visit to physician, emergency department).

Physical activity, balance and mobility: Self-reported frequency and duration of physical activity will be obtained using the Incidental and Planned Exercise Questionnaire. ${ }^{31}$ Physical activity levels and mobility during daily life activities will be monitored over 1 week using the McRoberts MoveMonitor (McRoberts, the Netherlands). ${ }^{32}$ Fall risk will be assessed using the Physiological Profile Assessment, which contains individual tests of knee extension strength, postural sway, a limb matching task, simple hand reaction time (RT) and the Melbourne-edge test of contrast sensitivity - not available for assessments via telehealth). Standing balance is assessed during different feet positions for a maximum of $30 \mathrm{~s}$ per condition (feet together, near-tandem, tandem and on left and right foot on floor, if any position was sustained for $\geq 15 \mathrm{~s}$, the position was repeated on a foam cushion). ${ }^{8}$ Controlled leaning balance is assessed using the maximum anteroposterior lean test and coordinated stability test ${ }^{8}$ - not available for assessments via telehealth), which requires participants to lean back and forth as far as they can and follow a butterfly shape by leaning their body without moving their feet. Mobility is assessed using the Short Physical Performance Battery, ${ }^{33}$ Timed Up-and-Go, ${ }^{34} 5$-times sit-to-stand, ${ }^{35} 10 \mathrm{~m}$ walk $\operatorname{test}^{36}(4 \mathrm{~m}$ walk test for assessments via telehealth) and $10 \mathrm{~m}$ walk test with a cognitive dual task of calling out every second letter of the alphabet ${ }^{37}$ — not available for assessments via telehealth). Participants are asked to perform the 5-times sit-to-stand as quickly as possible while staying safe; they are instructed to perform all other mobility tests at their habitual walking speed. Self-reported change in balance, physical activity and overall quality of life will be obtained using the Patient's Global Impression of Change Scale. ${ }^{38}$

Cognitive function: Executive functioning is assessed using the Cogstate Groton maze and one-back tests ${ }^{39}$ and trail making tests (conditions A and B) ${ }^{40}$ Processing speed is assessed using five RT tests (not available for assessments via telehealth): three stepping RT tests on an electronic step mat with six target panels (ie, Choice, Stroop and inhibitory go/no-go (ref Lord)); Cars choice RT test involving foot and hand responses ${ }^{41}$ and ReacStick 'catch-the-ruler' test under simple and inhibitory go/ no-go conditions. ${ }^{42}$

Psychological well-being, quality of life and health literacy: Depressive symptoms are assessed using the 15-item Geriatric Depression Scale ${ }^{43}$ and Depression, Anxiety and Stress Scales. ${ }^{44}$ Concern about falling is assessed using the iconographic Falls Efficacy Scale. ${ }^{45}$ Well-being is assessed using the 26-item COMPAS-W scale of wellbeing ${ }^{46}$ and the occurrence of major daily life events is recorded using the Daily Life Event scale. ${ }^{47}$ Quality of life is assessed using the 5-level European QoL-5 dimensions, ${ }^{48}$ WHO Disability Assessment Schedule ${ }^{49}$ and the ICEpop CAPability measure for Older people. ${ }^{50}$ Health literacy is assessed using the Health Literacy Questionnaire. $^{51}$

User experience: User experience will be assessed using the Physical Activity Enjoyment Scale, ${ }^{52}$ System Usability Scale, ${ }^{53}$ Attitudes to Falls Related Intervention Scale ${ }^{54}$ and Exercise Self-Efficacy Scale. ${ }^{55}$ 
Adherence, healthcare use and intervention costs: Adherence will be obtained from our customer management systems as weekly exercise minutes for StandingTall and StandingTall+Cognitive, as modules completed for myCompass, and as factsheets accessed for the health education promotion programme. Healthcare use will be obtained using a monthly questionnaire on use of health and community services, and on inpatient hospitalisations, ambulance calls, emergency department presentations, pharmaceutical prescriptions and medical services via data linkage with the Centre for Health Record Linkage. Intervention costs will be obtained from the project financial records and include resource and personnel costs (eg, purchase of iPads, 3/4G access, costs of RAs).

Adverse events: All adverse events (defined as any untoward medical occurrences that happen to a participant during the study), that are reported by the participant or observed by the investigators or study staff, will be recorded. Falls that do not involve study procedures or equipment will not be considered untoward and will not be included as adverse events. Given the relatively low risk of the trial, adverse events will be continuously reviewed, reported to the NeuRA's safety committee and UNSW's Ethics as appropriate, and discussed during yearly safety committee meetings (online supplemental file 2).

\section{Data analysis plan}

\section{Effectiveness analysis}

Analysis of effectiveness on the primary and secondary outcomes will use intention to treat, and will be blinded for intervention allocation by having a researcher not involved in the analyses recode the groups using two random numbers ( $\mathrm{eg}$, groups ' 1 ' and ' 2 ', instead of 'intervention' and 'control'). Our primary outcome will be compared between groups as the number of falls per person-year using Poisson or negative binomial regression (depending on the data distribution). In secondary analyses, complier averaged causal effect analysis will be used to correct for imperfect adherence, and the proportion of fallers will be compared between groups using modified Poisson or logistic regression (depending on the data distribution). The effect of group allocation on secondary outcomes will be analysed using generalised linear models. The effect of in-person versus telehealth assessment will be explored using a covariate accounting for method of assessment.

\section{Economic analysis}

Economic analysis will be conducted from a health and community care provider perspective and comprise a cost-effectiveness analysis and a cost-utility analysis. ${ }^{56}$ The analysis will include costs associated with intervention delivery and healthcare utilisation, and the outcomes of interest will be the number of falls prevented and healthrelated quality of life. Bootstrapping will be used to estimate a distribution around costs and health outcomes, and to calculate the confidence intervals around the incremental cost-effectiveness ratios. A cost-effectiveness acceptability curve will be plotted to provide information about the probability that the intervention is cost-effective at different willingness to pay thresholds.

\section{Data integrity and missingness}

Data will be regularly monitored for omissions and errors. All analyses will follow a detailed statistical analysis plan, which will be publicly registered prior to analysis of the data. We will not have to account for missing data in the primary outcome as a correction for follow-up duration is part of the analysis. Missing data in other outcomes will be imputed using estimated means or multiple imputation depending on the mechanism of missingness. In case of a large proportion of missing data, which is anticipated for some outcomes due to our switch to telehealth, we will report both imputed and complete cases results where appropriate.

\section{ETHICS AND DISSEMINATION}

Ethical approval has been obtained from the University of New South Wales Human Research Ethics Committee (HC17977). The study is considered to pose a low risk to participant safety. Intervention participants will engage in balance exercise, which poses a small risk for musculoskeletal complaints and accidental falls. These risks are considered inherent to physical activity, which generally provides more benefit than harm when practised in reasonable amounts. The switch to telehealth reduces accessibility to the study for older people without internet access. This issue is partially remediated by providing $3 / 4 \mathrm{G}$ connectivity to participants who do not have internet access.

Dissemination of study findings is planned via peerreviewed journals, national and international conferences, community events and media releases. Data will be available for secondary analyses by contacting the corresponding author. The results of this study will provide evidence towards a more effective and accessible multifactorial fall prevention programme for high-risk populations, by addressing physical, affective and cognitive fall risk factors. StandingTall + promotes active engagement by the older person and the use of technology allows tailoring without increasing complexity of the intervention. The use of technology will also allow rapid translation into scalable and sustainable improvements in clinical practice and patient outcomes. The cost-effectiveness evaluation will be a crucial step to convince health promotion practitioners and public health policy makers towards the broad implementation of our proposed model towards assigning people to certain tailored intervention strategies as part of routine healthcare.

\section{Author affiliations}

${ }^{1}$ Neuroscience Research Australia, Randwick, New South Wales, Australia ${ }^{2}$ School of Population Health, University of New South Wales, Sydney, New South Wales, Australia 
${ }^{3}$ Peninsula Clinical School, Central Clinical School, Monash University, Melbourne, Victoria, Australia

${ }^{4}$ Menzies Institute for Medical Research, University of Tasmania, Hobart, Tasmania, Australia

${ }^{5}$ The Black Dog Institute and School of Medicine, University of New South Wales, Sydney, New South Wales, Australia

${ }^{6}$ The George Institute for Global Health, University of New South Wales, Sydney, New South Wales, Australia

${ }^{7}$ School of Public Health, Faculty of Medicine and Health, University of Sydney, Sydney, New South Wales, Australia

${ }^{8}$ School of Psychology, University of New South Wales, Sydney, New South Wales, Australia

Contributors KD conceived the idea for the trial, and oversaw the development of the StandingTall and StandingTall+Cognitive intervention programmes, assisted by KSvS, MC, JC and GM. The trial was designed by KD, assisted by KSvS, MC, $\mathrm{BO}, \mathrm{SRL}, \mathrm{KJA}, \mathrm{HC}$ and implemented by JC, GM, LM, AB, AP and MN. The economic analyses were designed by TL. Funding was obtained by KD, KJA, MC, BO, SRL and $\mathrm{HC}$. KSvS and KD prepared the first draft of the manuscript. All authors contributed to the refinement of the study protocol and approved the final version of the manuscript.

Funding This work is supported by the Australian National Health and Medical Research Council (NHMRC, grant number APP1139673), KD is supported by an NHMRC Investigator grant, KSvS by an HFSP fellowship, and SRL through an NHMRC Senior Principal Research Fellowship.

Disclaimer The funding sources had no role in the design of this study and will not have a role during its execution, analyses, interpretation or decision to publish results.

Competing interests The physiological profile assessment is commercially available from Neuroscience Research Australia.

Patient consent for publication Not required.

Provenance and peer review Not commissioned; peer reviewed for ethical and funding approval prior to submission.

Supplemental material This content has been supplied by the author(s). It has not been vetted by BMJ Publishing Group Limited (BMJ) and may not have been peer-reviewed. Any opinions or recommendations discussed are solely those of the author(s) and are not endorsed by BMJ. BMJ disclaims all liability and responsibility arising from any reliance placed on the content. Where the content includes any translated material, BMJ does not warrant the accuracy and reliability of the translations (including but not limited to local regulations, clinical guidelines, terminology, drug names and drug dosages), and is not responsible for any error and/or omissions arising from translation and adaptation or otherwise.

Open access This is an open access article distributed in accordance with the Creative Commons Attribution Non Commercial (CC BY-NC 4.0) license, which permits others to distribute, remix, adapt, build upon this work non-commercially, and license their derivative works on different terms, provided the original work is properly cited, appropriate credit is given, any changes made indicated, and the use is non-commercial. See: http://creativecommons.org/licenses/by-nc/4.0/.

ORCID iDs

Thomas Lung http://orcid.org/0000-0001-9978-6311

Kim Delbaere http://orcid.org/0000-0002-5655-0234

\section{REFERENCES}

1 Franco MR, Tong A, Howard K, et al. Older people's perspectives on participation in physical activity: a systematic review and thematic synthesis of qualitative literature. Br J Sports Med 2015;49:1268-76.

2 Agmon M, Perry CK, Phelan E, et al. A pilot study of Wii fit exergames to improve balance in older adults. J Geriatr Phys Ther 2011;34:161-7.

3 Schoene D, Valenzuela T, Toson B, et al. Interactive CognitiveMotor Step Training Improves Cognitive Risk Factors of Falling in Older Adults - A Randomized Controlled Trial. PLoS One 2015;10:e0145161.

4 Delbaere K, Valenzuela T, Lord S. e-Health "StandingTall" balance exercise for fall prevention in older people: Results of a two-year randomised controlled trial. $B M J$.
5 Martin KL, Blizzard L, Wood AG, et al. Cognitive function, gait, and gait variability in older people: a population-based study. J Gerontol A Biol Sci Med Sci 2013;68:726-32.

6 Kvelde T, Lord SR, Close JCT, et al. Depressive symptoms increase fall risk in older people, independent of antidepressant use, and reduced executive and physical functioning. Arch Gerontol Geriatr 2015;60:190-5.

7 van Schooten KS, Taylor ME, Close JCT, et al. Sensorimotor, cognitive, and affective functions contribute to the prediction of falls in old age and neurologic disorders: an observational study. Arch Phys Med Rehabil 2020. doi:10.1016/j.apmr.2020.10.134. [Epub ahead of print: 27 Nov 2020].

8 Lord SR, Menz HB, Tiedemann A. A physiological profile approach to falls risk assessment and prevention. Phys Ther 2003;83:237-52. doi:10.1093/ptj/83.3.237

9 Delbaere K, Close JCT, Heim J, et al. A multifactorial approach to understanding fall risk in older people. J Am Geriatr Soc 2010;58:1679-85.

10 Callisaya ML, Ayers E, Barzilai N, et al. Motoric cognitive risk syndrome and falls risk: a multi-center study. J Alzheimers Dis 2016;53:1043-52.

11 Delbaere K, Kochan NA, Close JCT, et al. Mild cognitive impairment as a predictor of falls in community-dwelling older people. Am J Geriatr Psychiatry 2012;20:845-53.

12 Eysenck MW, Derakshan N, Santos R, et al. Anxiety and cognitive performance: attentional control theory. Emotion 2007;7:336-53.

13 Anstey KJ, Burns R, von Sanden C, et al. Psychological well-being is an independent predictor of falling in an 8-year follow-up of older adults. J Gerontol B Psychol Sci Soc Sci 2008;63:P249-57.

14 Hopewell S, Adedire O, Copsey BJ, et al. Multifactorial and multiple component interventions for preventing falls in older people living in the community. Cochrane Database Syst Rev 2018;7:CD012221.

15 Zijlstra GAR, van Haastregt JCM, Ambergen T, et al. Effects of a multicomponent cognitive behavioral group intervention on fear of falling and activity avoidance in community-dwelling older adults: results of a randomized controlled trial. J Am Geriatr Soc 2009;57:2020-8.

16 Mirelman A, Rochester L, Maidan I, et al. Addition of a nonimmersive virtual reality component to treadmill training to reduce fall risk in older adults (V-TIME): a randomised controlled trial. Lancet 2016;388:1170-82

17 Campbell AJ, Robertson MC. Rethinking individual and community fall prevention strategies: a meta-regression comparing single and multifactorial interventions. Age Ageing 2007;36:656-62.

18 Pfeiffer E. A short portable mental status questionnaire for the assessment of organic brain deficit in elderly patients. J Am Geriatr Soc 1975;23:433-41.

19 Kroenke K, Spitzer RL, Williams JB. The PHQ-9: validity of a brief depression severity measure. J Gen Intern Med 2001;16:606-13.

20 Hile ES, Brach JS, Perera S, et al. Interpreting the need for initial support to perform tandem stance tests of balance. Phys Ther 2012;92:1316-28.

21 Proudfoot J, Clarke J, Birch M-R, et al. Impact of a mobile phone and web program on symptom and functional outcomes for people with mild-to-moderate depression, anxiety and stress: a randomised controlled trial. BMC Psychiatry 2013;13:312.

22 Haran MJ, Cameron ID, Ivers RQ, et al. Effect on falls of providing single lens distance vision glasses to multifocal glasses wearers: visible randomised controlled trial. $B M J$ 2010;340:1345:c2265.

23 Franco MR, Howard K, Sherrington C, et al. Smallest worthwhile effect of exercise programs to prevent falls among older people: estimates from benefit-harm trade-off and discrete choice methods. Age Ageing 2016;45:806-12.

24 Sherrington C, Michaleff ZA, Fairhall N, et al. Exercise to prevent falls in older adults: an updated systematic review and meta-analysis. $\mathrm{Br}$ $J$ Sports Med 2017;51:1750-8.

25 Gillespie LD, Robertson MC, Gillespie WJ, et al. Interventions for preventing falls in older people living in the community. Cochrane Database Syst Rev 2012;2:CD007146.

26 Lamb SE, Jørstad-Stein EC, Hauer K, et al. Development of a common outcome data set for fall injury prevention trials: the prevention of falls network Europe consensus. J Am Geriatr Soc 2005:53:1618-22.

27 Charlson ME, Pompei P, Ales KL, et al. A new method of classifying prognostic comorbidity in longitudinal studies: development and validation. J Chronic Dis 1987;40:373-83.

28 Costa P, McCrae R. NEO inventories professional manual. Psychological Assessment Resources, Inc, 2010. 
29 Nasreddine ZS, Phillips NA, Bédirian V, et al. The Montreal cognitive assessment, MoCA: a brief screening tool for mild cognitive impairment. J Am Geriatr Soc 2005;53:695-9.

30 Baddeley A, Emslie H, Nimmo-Smith I. The Spot-the-Word test: robust estimate of verbal intelligence based on lexical decision. $\mathrm{Br} \mathrm{J}$ Clin Psychol 1993;32:55-65.

31 Delbaere K, Hauer K, Lord SR. Evaluation of the incidental and planned activity questionnaire (IPEQ) for older people. Br J Sports Med 2010;44:1029-34.

32 van Schooten KS, Pijnappels M, Rispens SM, et al. Daily-life gait quality as predictor of falls in older people: a 1-year prospective cohort study. PLoS One 2016;11:e0158623.

33 Guralnik JM, Simonsick EM, Ferrucci L, et al. A short physical performance battery assessing lower extremity function: association with self-reported disability and prediction of mortality and nursing home admission. J Gerontol 1994;49:M85-94.

34 Podsiadlo D, Richardson S. The timed "Up \& Go": a test of basic functional mobility for frail elderly persons. J Am Geriatr Soc 1991;39:142-8

35 Csuka M, McCarty DJ. Simple method for measurement of lower extremity muscle strength. Am J Med 1985;78:77-81.

36 Bohannon RW. Comfortable and maximum walking speed of adults aged 20-79 years: reference values and determinants. Age Ageing 1997;26:15-19.

37 Brandler TC, Oh-Park M, Wang C, et al. Walking while talking: investigation of alternate forms. Gait Posture 2012;35:164-6.

38 Guy W. ECDEU assessment manual for psychopharmacology: US department of health, education and welfare, public health service, 1976.

39 Mielke MM, Machulda MM, Hagen CE, et al. Performance of the CogState computerized battery in the Mayo Clinic Study on Aging. Alzheimers Dement 2015;11:1367-76.

40 Reitan RM, Wolfson D. The Halstead-Reitan neuropsychological test battery: theory and clinical interpretation. Tucson, Ariz: Neuropsychology Press, 1985.

41 Anstey KJ, Wood J, Kerr G, et al. Different cognitive profiles for single compared with recurrent fallers without dementia. Neuropsychology 2009;23:500-8.

42 van Schooten KS, Duran L, Visschedijk M, et al. Catch the ruler: concurrent validity and test-retest reliability of the ReacStick measures of reaction time and inhibitory executive function in older people. Aging Clin Exp Res 2019;31:1147-54.
43 Sheikh JI, Yesavage JA, Scale GD. Geriatric depression scale (GDS): recent evidence and development of a shorter version. Clin Gerontol 1986. doi:10.1300/J018v05n01_09

44 Lovibond SH, Lovibond PF. Manual for the depression anxiety stress scales: psychology Foundation of Australia, 1996.

45 Delbaere K, Smith ST, Lord SR. Development and initial validation of the Iconographical falls efficacy scale. J Gerontol A Biol Sci Med Sci 2011;66:674-80.

46 Gatt JM, Burton KLO, Schofield PR, et al. The heritability of menta health and wellbeing defined using COMPAS-W, a new composite measure of wellbeing. Psychiatry Res 2014;219:204-13.

47 Gatt JM, Korgaonkar MS, Schofield PR, et al. The TWIN-E project in emotional wellbeing: study protocol and preliminary heritability results across four MRI and DTI measures. Twin Res Hum Genet 2012;15:419-41.

48 Viney R, Norman R, King MT, et al. Time trade-off derived EQ-5D weights for Australia. Value Health 2011;14:928-36.

49 Ustün TB, Chatterji S, Kostanjsek N, et al. Developing the world Health organization disability assessment schedule 2.0. Bull World Health Organ 2010;88:815-23.

50 Grewal I, Lewis J, Flynn T, et al. Developing attributes for a generic quality of life measure for older people: preferences or capabilities? Soc Sci Med 2006;62:1891-901.

51 Osborne RH, Batterham RW, Elsworth GR, et al. The grounded psychometric development and initial validation of the health literacy questionnaire (HLQ). BMC Public Health 2013;13:658.

52 Kendzierski D, DeCarlo KJ. Physical activity enjoyment scale: two validation studies. J Sport Exerc Psychol 1991;13:50-64.

53 Bangor A, Kortum P, Miller J. Determining what individual Sus scores mean: adding an adjective rating scale. J Usability Stud 2009;4:114-23.

54 Yardley L, Donovan-Hall M, Francis K, et al. Attitudes and Beliefs That Predict Older People's Intention to Undertake Strength and Balance Training. J Gerontol B Psychol Sci Soc Sci 2007;62:P119-25.

55 Everett B, Salamonson Y, Davidson PM. Bandura's exercise selfefficacy scale: validation in an Australian cardiac rehabilitation setting. Int J Nurs Stud 2009;46:824-9.

56 Davis JC, Robertson MC, Comans T, et al. Guidelines for conducting and reporting economic evaluation of fall prevention strategies. Osteoporos Int 2011;22:2449-59. 